\title{
Dispositifs de groupware : entre prescriptions et usages réels
}

\section{Valérie Lépine}

\section{(2) OpenEdition}

1 Journals

Édition électronique

URL : http://journals.openedition.org/communicationorganisation/2485

DOI : 10.4000/communicationorganisation.2485

ISSN : $1775-3546$

Éditeur

Presses universitaires de Bordeaux

Édition imprimée

Date de publication : 1 mai 2001

ISSN : 1168-5549

Référence électronique

Valérie Lépine, «Dispositifs de groupware : entre prescriptions et usages réels », Communication et organisation [En ligne], 19 | 2001, mis en ligne le 27 mars 2012, consulté le 20 avril 2019. URL : http:// journals.openedition.org/communicationorganisation/2485; DOI : 10.4000/ communicationorganisation. 2485

Ce document a été généré automatiquement le 20 avril 2019.

(c) Presses universitaires de Bordeaux 


\title{
Dispositifs de groupware : entre prescriptions et usages réels
}

\author{
Valérie Lépine
}

1 Dès la fin des années 80 dans les pays anglo-saxons et au milieu des années 90 en France les responsables informatiques des entreprises ont marqué leur intérêt pour un logiciel, Lotus Notes, racheté et promu par IBM depuis 1996. Ce produit commercial, associé au concept de groupware, fut alors présenté comme une petite révolution du système d'information et de communication : les entreprises allaient entrer dans l'ère du travail coopératif. La coopération, le partage d'informations, les communications distantes et asynchrones entre équipes distribuées, le travail en réseau sont autant de notions qui se sont superposées à celle de groupware sans qu'une définition consensuelle ou un périmètre technique circonscrit aient $\mathrm{pu}$ y être attachés. Le succès du groupware s'explique par l'intervention d'un certain nombre d'acteurs qui ont eu intérêt au développement de ce nouveau marché. Mais il a surtout été conditionné par l'adhésion des décideurs aux promesses de la coopération; promesses qui ont pris la forme d'une véritable injonction collaborative auprès des agents dans l'entreprise. De quelle coopération s'agit-il en réalité ? Y a-t-il d'autres finalités objectives qui guident les entreprises vers le déploiement de dispositifs de groupware? Qu'advient-il des communications de travail lorsqu'elles sont médiatisées par un dispositif technique?

\section{Le cadre de la recherche}

2 Nous proposons dans cet article de présenter une synthèse partielle de l'étude empirique de trois cas d'entreprises ayant choisi d'implanter des dispositifs de groupware et qui constitue l'un des versants d'une recherche plus globale menée dans le cadre de la réalisation d'une thèse de doctorat en Sciences de la Communication (Lépine. 2000). Propose aux décideurs du marché et promu comme un concept à la croisée de l'informatique du management et de l'organisation (Levan \& Liebman, 1996), le groupware ne s'inscrit pas seulement dans la continuité des évolutions des NTIC, il perpétue et prolonge une réflexion sur le rôle et la nature de la coopération dans l'organisation d'une 
part, sur son contrôle et sa gestion par le management d'autre part. La notion de coopération est le fruit d'une progressive élaboration dans la pensée managériale et gestionnaire (Lépine, 1999), elle est aussi polysémique car elle est attachée à des représentations et à des croyances qui concernent à la fois la division et l'organisation du travail, la construction de buts ou d'objectifs partagés et le sens qui leur est donné par les individus (Zarifian, 1996 ; Linhart, 1994). Aussi est-il nécessaire de resituer l'analyse des déploiements de groupware en entreprise dans le cadre plus large des dynamiques sociales, organisationnelles et symboliques qui structurent et conditionnent leur mise en œuvre et leur appropriation dans les usages professionnels.

Les résultats présentés dans cet article sont issus d'une investigation comparative menée dans trois entreprises du secteur privé marchand qui ont pour point commun l'adoption du logiciel de groupware Lotus Notes. La méthodologie, qualitative, repose sur la conduite, sur le lieu de travail, de vingt-huit entretiens semi-directifs complétés par des observations sur l'utilisation des applications de groupware. Les entreprises retenues présentent des profils volontairement contrastés : le premier cas est celui d'une petite start-up régionale (Isys) spécialisée dans la prestation de services informatiques précisément sous Lotus Notes; le second présente les enjeux d'une des entités industrielles (Ugine) d'un grand groupe français de sidérurgie en phase de restructuration et de refonte de son système d'information; le troisième cas est celui d'un des grands cabinets d'audit anglo-saxons de dimension mondiale (Ernst \& Young) ayant décidé en 1994 de déployer Lotus Notes dans l'ensemble du groupe au niveau mondial.

Nous avons cherché à vérifier par l'enquête de terrain l'hypothèse initiale selon laquelle le groupware pourrait être l'indice, le prétexte ou l'instrument d'un processus de rationalisation du travail intellectuel et notamment des cadres (inscrit dans la continuité historique de la rationalisation du travail depuis l'émergence de l'entreprise moderne). Deux autres hypothèses complètent cette perspective voisine des approches de la sociologie du monde du travail et qui orientent la problématique vers un point de vue propre aux sciences de la communication. D'une part, la coopération promue par les prescripteurs de ce nouveau marché serait fortement cadrée, techniquement, organisationnellement, mais aussi symboliquement, dans la mesure où elle est accompagnée de discours et de pratiques qui touchent le statut et la signification accordés aux échanges et aux communications de travail. D'autre part, le déploiement d'un projet de groupware pourrait offrir l'opportunité d'une plus grande maîtrise de la communication de/au travail avec une visée d'accroissement de la productivité mais peut-être aussi avec une finalité de contrôle accru des relations sociales dans l'entreprise.

\section{La rationalisation des processus de travail intellectuel : une réalité contrastée et des problématiques différenciées}

5 La préoccupation de la rationalisation des modes d'organisation du travail est à la fois ancienne et au fondement même de la pensée gestionnaire. Elle est puissamment réactivée par les enjeux de la mondialisation des échanges et renouvelée dans sa nature, en liaison avec l'importance prise par le facteur informationnel dans les systèmes économiques et productifs occidentaux. Dans les entreprises, la poursuite des objectifs de modernisation et de renforcement de l'efficience productive a favorisé le développement 
massif de systèmes d'information fortement technicisés, pour la production la logistique ou la gestion des ventes, c'est à dire les fonctions étroitement liées au processus industriel qui va de la conception à la mise en marché des produits. La phase d'informatisation liée à la mise en œuvre de groupware vise un autre niveau de l'organisation : celui de la gestion de projets, des tâches de coordination, de planification, de redistribution et d'ajustement des activités qui incombe au management.

Le groupware a pour objectif explicite l'optimisation des missions fonctionnelles, administratives et organisationnelles accomplies pour l'encadrement d'équipes de travail. L'étude du déploiement de tels dispositifs dans des entreprises très différenciées permet d'apporter des éléments empiriques et d'apprécier, au moins qualitativement, la portée de l'objectif d'efficience productive proposée par le groupware, qui est inscrite dans le processus historique plus global de la rationalisation du travail. La réalité des problématiques et des enjeux identifiés dans les trois cas étudiés s'avère contrastée, et conduit à nuancer ou à reformuler cette hypothèse de départ.

\section{Le cas ISYS : une co-construction des dimensions techniques, organisationnelles et sociales plutôt qu'une rationalisation}

7 Dans la petite "start up " dédiée au mono-produit Lotus Notes, le système technique d'information et de communication entre les membres de l'entreprise se superpose à l'outil de travail d'une part, à la mise en place de l'organisation administrative et commerciale d'autre part. En effet, le collecticiel Lotus Notes a été originellement adopté dès la création de la structure ISYS, pour en tester les potentialités et en explorer les fonctionnalités, avant le développement d'applications commerciales pour les premiers clients. L'étude montre l'étroite imbrication des ajustements successifs entre le système d'information, les usages concrets et les évolutions des différentes applications, l'organisation du travail et les relations d'échanges et de communications internes et externes. Il n'y a pas. dans cette entreprise naissante, une problématique de changement technique ou organisationnel, mais une logique de co-émergence et de co-structuration d'une entreprise s'instituant autour d'un projet commun et d'intérêts individuels, d'une organisation où se définissent des relations de travail, des rôles, des fonctions, des manières de travailler toujours en évolution, et d'un système d'information et de communication qui reflète, cristallise et amplifie les composantes précédentes.

$8 \quad$ La SSII est composée uniquement de personnel hautement qualifié travaillant sur des projets de conception et de commercialisation de services qui mobilisent constamment des qualités d'interprétation, d'analyse et de décision, propres au travail intellectuel. Pour autant, dans cette entreprise il serait erroné de formuler les enjeux du système d'information dans les termes de la rationalisation des processus du travail intellectuel. Les applications développées correspondent davantage à l'émergence et à la consolidation d'une organisation où chacun trouve avantage à adopter le système de groupware: telle secrétaire administrative aura intérêt à reporter sur le dispositif technique la gestion des frais, des facturations, du planning afin de consacrer le temps gagné à une évolution professionnelle souhaitée vers des fonctions commerciales plus valorisantes; les développeurs trouveront un bénéfice réel à partager des connaissances sur la résolution de problèmes, etc. 


\section{UGINE des enjeux de normalisation-standardisation}

9 Le cas UGINE révèle des logiques complexes, qui mettent en rapport des enjeux de modernisation et de compétitivité d'un groupe international avec ceux de l'amélioration de la productivité de la part informationnelle du système productif. Cette part informationnelle de production de la valeur, et/ou du produit final lui-même, prend préférentiellement place dans les activités de recherche, de méthode, d'organisation et de coordination.

10 Réalisée dans une entité régionale, la recherche a mis l'accent sur l'importance du rôle intégrateur et normatif d'une nouvelle organisation par marché décidée par le siège parisien ainsi que du projet de refonte globale du système d'information du groupe. La création de la fonction du responsable groupware et le discret encouragement de la mise en place d'applications, principalement dans les services fonctionnels administratifs ou de support transversal à la production (qualité, méthodes, travaux informatiques, essais et recherche, gestion financière et gestion des ressources humaines), répondent certes à des impératifs de meilleure synergie entre les unités fonctionnelles d'entreprises historiquement distinctes, souvent concurrentes avant les restructurations. Mais, au-delà des enjeux d'efficience organisationnelle, cette étude de cas montre l'importance cruciale de l'enjeu de normalisation symbolique et communicationnelle qui sous-tend la standardisation des outils informationnels à travers les dispositifs informatiques. Ainsi une application développée par le service des méthodes permet-elle d'imposer aux ouvriers des usines des nomenclatures et un lexique liés aux produits sidérurgiques dans un langage qui ignore totalement les parlers traditionnels du métier ou des régions; ce faisant, elle dénie la culture et les savoirs ouvriers en ne retenant comme légitimes que les seuls savoirs du bureau des méthodes, détenteur de la maitrise tant de la forme que des contenus des applications informatiques.

11 Tandis que d'autres travaux en gestion ou management posent la question du lien entre système technique et changements organisationnels (Fulk \& Desanctis, 1995) l'approche communicationnelle met l'accent sur les dimensions plus directement liées à la relation (donc aux relations sociales) et aux enjeux de la médiatisation technique des activités informationnelles et des communications de travail. En regard de l'hypothèse d'une rationalisation du travail intellectuel, cette perspective conduit, ici encore, à une reformulation des termes de la problématique, en posant la question du déplacement des instruments de standardisation-normalisation de l'outil de production industriel vers ses activités informationnelles de support.

\section{ERNST \& YOUNG un cas où se vérifie l'hypothèse de la rationalisation du travail intellectuel}

12 C'est seulement dans la troisième entreprise étudiée que la question de la rationalisation du travail intellectuel est patente dans le déploiement massif du système d'information et de communication ainsi que dans la politique, souhaitée et mise en place par la direction, des outils, des règles et des principes du travail de groupe. L'analyse de cette politique de rationalisation conduit à la conclusion du renforcement de la productivité de l'activité intellectuelle à proprement parler, en particulier par l'intensification de l'activité des personnels de ce grand cabinet d'audit et de conseil. Elle se traduit à la fois par une 
densification du travail à haute valeur ajoutée : certaines tâches annexes sont évitées ou rendues plus rapides grâce à l'utilisation du système groupware (recherche d'informations, et copie ou transfert de documents) ; tandis que le transfert d'activités organisationnelles ou fonctionnelles des personnels administratifs vers les consultants (saisie des temps passés et des frais, organisation de réunions via l'agenda électronique, traitement des mails), génère un surcroît de travail effectué en dehors du temps de production intellectuelle, prenant le pas sur l'espace de vie privée et sur le temps de loisirs.

La rationalisation de l'activité de production de services immatériels est aussi renforcée par le processus de normalisation-standardisation des tâches, évoqué supra, et qui concerne ici le processus même de la démarche intellectuelle au cœur de l'activité des consultants. En effet, en particulier dans les métiers du chiffre (audit, expertise comptable), la volonté de la direction du groupe de revaloriser une activité, très concurrencée et en voie de banalisation, a abouti à la mise en place de dispositifs informatisés susceptibles de normaliser fortement les méthodes d'audit et de comptabilité (documents électroniques préformatés et workflow de constitution et de validation de toutes les étapes de traitement des dossiers), là où existait auparavant une plus grande diversité des pratiques liées à l'expertise et au jugement des consultants.

Pour autant et bien que le déploiement des outils informatiques ait été rapide, massif et relativement autoritaire via la création de "passages obligés » - volonté du zéro papier pour tous les formulaires administratifs, utilisation obligatoire de la messagerie électronique pour l'organisation de réunions ou pour l'accès aux messages téléphoniques externes -, une analyse plus politique des enjeux et des stratégies montre aussi qu'il n'y a ni déterminisme technique direct ni machiavélisme social dans un tel processus. Un des facteurs explicatifs de la politique de rationalisation et de recherche des zones de plus grande productivité technico-organisationnelle est d'ordre politique, et renvoie à la concentration des pouvoirs financiers et décisionnels (Pichault, 1995) entre les mains des seuls associés, dont la rémunération est directement issue des marges réalisées sur le travail des consultants. Un autre facteur explicatif des modes d'appropriation ou des comportements de contournement de la politique de changement technologique, est lié à la structure organisationnelle et à la culture d'entreprise (Orlikowski. 1992) qui favorise un fort individualisme professionnel et la préservation par les consultants de leur zone d'expertise personnelle.

\section{Groupware et coopération : qu'en est-il dans les faits ?}

La seconde hypothèse est celle d'une coopération en réalité « cadrée » par la technique, c'est à dire par la conception même du logiciel et de ses fonctionnalités, par l'organisation et l'ensemble des facteurs sociaux liés à la division du travail, aux rapports hiérarchiques et sociaux, et enfin, cadrée symboliquement par l'injonction faite aux salariés de coopérer et par les représentations légitimes attachées à cette injonction. Appréhender le dispositif de groupware dans ces trois dimensions permet, nous semble-t-il, de ne pas réduire l'analyse à une évaluation proprement instrumentale des systèmes techniques, mais de saisir plus finement leur imbrication avec le corps social où ils prennent place. 


\section{ISYS : une dynamique « collaborative »}

16 Le cas ISYS montre que dans un "contexte socio-organisationnel » flexible et peu hiérarchisé, culturellement acquis à l'utilisation des systèmes techniques, la mise en place d'un dispositif de groupware puisse favoriser l'accès partagé à des informations construites en commun, supportant les pratiques de coopération existantes (partage de procédures de résolution de problèmes, bases de questions-réponses), et puisse aussi permettre l'émergence de nouvelles coopérations au méta-niveau de l'organisation (codéfinition de l'organisation du travail et co-construction des outils de support à la nouvelle organisation).

Du point de vue de la problématique de mise en rapport des systèmes de groupware et de la coopération dans le travail, cette étude de cas met en lumière la dynamique véritablement collaborative existant dans l'entreprise. Pour autant, on ne peut pas conclure à «l'impact» du seul dispositif informationnel, tant la force des interactions directes, d'une part, et des représentations mentales -individuelles et collectives- d'autre part, prédisposent les membres de l'équipe à une collaboration étroite et pleinement consentie. Cependant l'étude fait aussi apparaitre un effet de démultiplication ou d'amplification de cette dynamique collaborative, précisément à travers la médiatisation technique d'une partie des communications: envoi de plaisanteries et échanges conviviaux par le biais des forums ou de la messagerie, ou même à l'occasion de la transmission de données commerciales ou administratives : invitation à des rencontres informelles et festives par la voie de l'agenda électronique qui est aussi un outil de meilleure gestion du temps de travail, etc. Il y a donc une imbrication extrêmement forte entre les dimensions techniques, organisationnelles et sociales, visible dans la perméabilité du dispositif de groupware aux dynamiques proprement humaines.

De tels résultats évoquent les travaux du courant américain du constructivisme social des technologies de communication (Fulk, 1993) qui montrent que l'attitude individuelle à l'égard des techniques est fortement influencée par celle du groupe de travail: plus le groupe est favorable à ces outils plus l'individu adoptera une attitude positive, d'autant plus que sa motivation d'intégration au groupe est elle-même importante. Cette influence ne s'exerce pas seulement sur les pratiques d'utilisation (fréquence, utilité perçue des fonctionnalités), mais aussi sur "le sens donné au média » et à la perception de sa « richesse» qui sont socialement construits par le groupe de travail plus qu'ils ne sont objectivement déterminés par les utilités techniques réelles de l'objet.

\section{UGINE : une logique de « coordination »}

Dans cette entité industrielle nous avons indiqué l'importance primordiale du rôle intégrateur et normalisateur du système d'information. Beaucoup d'applications Lotus Notes n'ont du groupware que le nom, et ont été d'abord mises en place soit pour mettre à la disposition des intéressés des informations plutôt «statiques" (ex. les documents et procédures Qualité), soit pour mieux réguler et coordonner les activités d'un service sollicité par de nombreuses demandes extérieures (ex. les requêtes de travaux informatiques ou d'essais métallurgiques), soit enfin pour établir un cadre de saisie homogène et rapide de données issues de sites dispersés géographiquement (ex. la saisie du Bilan Social). Il s'agit dans la plupart des cas de formaliser et optimiser - au sens de 
rendre plus systématiques, plus rigoureux et plus visibles - des processus qui existaient auparavant sur papier ou disquettes informatiques, sans modification sensible de l'organisation du travail, tant au niveau des tâches individuelles qu'à celui du fonctionnement global du service. Le rôle du dispositif technique n'est pas précisément de développer ou d'encourager de nouvelles façons de travailler ensemble, mais d'opérer une médiation, de mettre en place des filtres fonctionnels, qui ont pour effet de limiter les sollicitations directes inopportunes pour l'organisation du service. Ces observations rejoignent celles de B. Faguet-Picq (1999), qui a étudié le cas de la mise en place d'un dispositif de groupware alimentant une hot-line technique (service d'assistance téléphonique) pour des commerciaux dispersés sur le territoire français, montrant que parfois le système technique "évite la dimension intersubjective du travail collectif », dans la mesure où « le dispositif opère les coordinations nécessaires ». La logique de mise en œuvre du groupware comme médiateur technique est, chez UGINE, celle d'une coordination mieux maîtrisée des activités de support, au prix d'une mise à distance de la communication directe dans certains cas.

\section{ERNST \& YOUNG : des résistances à l'égard d'une « coopération instrumentale»}

Ce cas ouvre la réflexion sur les formes de management de la coopération et sur l'instrumentation $\mathrm{du}$ partage des connaissances. Dans cette entreprise assez représentative $d u$ fonctionnement du secteur d'activité du conseil et de l'audit le déploiement mondial d'un système d'information et de communication groupware répond à un choix stratégique et managérial. Il s'agissait alors de saisir plus directement la nature du rapport entre le "projet groupware» en cours et les évolutions socioorganisationnelles vécues par les membres de l'organisation. En ce sens, ce dernier cas qui se veut exemplaire de la mise en œuvre d'un dispositif de travail coopératif global (et même globalisant) - est particulièrement révélateur des contradictions qui caractérisent les discours et les réalisations en matière de « management technologique ».

Le secteur de l'audit - comme beaucoup d'autres - est soumis à une pression concurrentielle croissante, de plus en plus internationale et étroitement liée à la capacité de valorisation rapide de ressources informationnelles dispersées aux seins d'entités géographiquement éloignées; et la plupart des grands acteurs du secteur ont dû adapter leur SI à ce contexte. Chez E \& Y, cette politique de changement technologique a été conduite avec une rapidité et un volontarisme exacerbés par le retard pris sur certains concurrents précurseurs. L'urgence ou la maladresse ne sont cependant pas les seuls facteurs explicatifs du profond décalage constaté entre, d'une part, les valeurs promues et les moyens mis au service de cet engagement entrepreneurial dans le travail coopératif et, d'autre part, la réalité des pratiques professionnelles et de la motivation individuelle à l'égard de l'injonction collaborative. L'explication d'une utilisation relativement distanciée des bases de discussion et de connaissances doit plutôt être trouvée en partie dans le système de pouvoir et de reconnaissance qui freine la contribution des consultants, qui n'ont aucun intérêt à partager leur expertise ni à s'exposer au jugement de leurs pairs ou de leurs supérieurs par le biais d'un dispositif technique qui n'est pas reconnu comme lieu légitime et équitable d'évaluation pour la progression de carrière.

Par ailleurs, l'extension des outils et des processus de médiation technique à des sphères de plus en plus nombreuses de l'activité professionnelle (gestion du temps, recherche 
d'informations, suivi des projets et des dossiers clients, etc.) est à la fois vécue comme un bénéfice, en termes d'organisation personnelle et collective (rapidité, praticité, traçabilité et fiabilité du travail informationnel), et comme une forte limitation de la subjectivité créative du travailleur intellectuel, d'une part, de l'inter-subjectivité constructive du groupe de travail ou de la relation de conseil aux clients, sur le terrain de l'action professionnelle et sans médiation, d'autre part. Or ce sont précisément ces dimensions de l'engagement subjectif et intersubjectif qui fondent la réelle nature de la collaboration, faute de quoi la coopération demeure purement fonctionnelle. Cela est vrai tant au niveau des cadres qu'à celui des personnels administratifs de la base (les secrétaires), dont les attitudes et les discours révèlent le primat, dans l'arbitrage des formes de l'interrelation, d'un contact direct avec les consultants qui permet un échange de plus grande qualité au plan de la relation humaine comme au plan de l'efficacité du transfert d'informations, là où l'entreprise préconise l'usage exclusif d'une application logicielle spécifique.

Il y a donc un antagonisme entre la logique gestionnaire qui souhaite imposer des outils de coopération fonctionnelle et des agents qui revendiquent la part incontournable du jugement personnel et de l'interrelation non médiée. Cet antagonisme ne doit pas masquer cependant l'existence d'un rapport de domination qui est en faveur de la première.

\section{L'hypothèse d'une maîtrise et d'un contrôle accrus des communications de/au travail}

Nous proposons de considérer ici des résultats qui portent, non pas sur la bonne ou la mauvaise gestion des flux d'échanges attendue des dispositifs de groupware, mais sur les dimensions communicationnelles des significations attribuées ou des contenus informationnels échangés par la médiation de ces dispositifs, ainsi que sur la dimension pragmatique de la relation telle qu'elle s'instaure dans - ou est cadrée par - l'usage et les représentations associées à l'outil de groupware.

Cette perspective nous conduit au constat d'un processus en œuvre, que nous qualifions de "gestion communicationnelle", qui est d'ampleur difficilement mesurable, mais qualitativement identifiable et lié à l'usage de plus en plus incontournable des objets de médiation technique des communications de travail. Le terme de gestion est à comprendre dans le sens d'une tendance à une plus rigoureuse administration des échanges, ce qui ne signifie pas (ni n'exclut pas) une économie ou une raréfaction de ces échanges. Nous analysons cette "gestion communicationnelle» à partir de trois dimensions de l'interaction médiatisée par un système technique de groupware: la gestion du flux des échanges et des activités de recherche d'informations; l'articulation du sens et de la forme des messages; la régulation de la relation interindividuelle ou entre l'individu et le collectif.

\section{La maîtrise des flux d'information et de communication :}

Il est probable que des études quantitatives montreraient l'augmentation significative, ces dernières années, du temps passé à traiter des informations par le biais du poste de travail informatisé. La grande majorité des personnes interviewées souligne, en même temps que la rapidité et la praticité de tels systèmes, les limites qu'il devient nécessaire 
de mettre en place pour faire face au «bruit » et au temps de traitement des informations qui arrivent quotidiennement. La maîtrise quantitative des flux peut donc être faite à l'initiative des utilisateurs, et opérée sous la forme d'une sélection des messages qui seront ou non traités; mais elle peut aussi faire l'objet d'un contrôle extérieur à l'utilisateur, voire être effectuée à son insu. En effet, il est très facilement possible d'avoir connaissance des sites Internet ou Intranet consultés sur chaque poste de travail, du temps et des fréquences de connexions. Chez E \& Y, la décision a été prise de restreindre les accès à l'Internet, et les consultants qui souhaitent en avoir l'usage doivent en faire une demande motivée, en sachant qu'une utilisation à titre privé serait rapidement identifiée. De même, les contributions aux forums et aux bases de connaissances sont aisément repérables et pourront être comptabilisées dans le système d'évaluation des personnels. La traçabilité des informations et des communications vient non seulement de la capacité d'organisation de stockage et de recherche incluse dans ces logiciels, mais elle porte aussi sur l'activité informationnelle et communicationnelle propre à chacun.

Si cette thématique n'est pas nouvelle (certaines entreprises annonçant par exemple le contrôle possible des numéros de téléphone appelés pour dissuader les utilisations privées), elle se renouvelle avec des technologies informatiques conçues, dès le départ, pour permettre facilement de tels contrôles sans surcoût et sans reprogrammation.

\section{Une économie du sens et de la forme des écrits électroniques :}

De façon récurrente, et quelle que soit l'entreprise considérée, les utilisateurs de messagerie ou de forums évoquent la spécificité de cette modalité particulière de correspondance, celle d'un écrit au statut ambigu qui n'a ni le statut officiel des écrits traditionnels de travail, ni la richesse (et la volatilité) des paroles échangées lors d'interactions directes de face-à-face. On peut interroger la validité des discours des salariés qui croient percevoir une substitution des communications orales directes par des échanges électroniques. Cette perception peut être quantitativement erronée et nous avons fait le constat contradictoire de la prédominance des échanges oraux, et même de leur sensible augmentation, entre des populations éloignées, qui ont pu entrer en contact grâce aux réseaux de communication et maintenir ensuite d'autres formes d'échanges plus directes. Par ailleurs, si certains renâclent à juste titre à l'usage des outils électroniques lorsque la situation (de négociation, de conflit ou bien de convivialité et de recherche de mise en confiance) justifie une communication réellement interactive de face-à-face, on peut s'interroger sur le fondement réel des jugements portés sur la " pauvreté » intrinsèque du média électronique.

Autrement dit, l'appréciation de la qualité de la communication semble devoir être liée à la force et à la légitimité de l'orientation d'un cadre communicationnel global, plutôt qu'aux caractéristiques de telle ou telle forme (ou médiation) de l'échange. Dans les organisations observées, il apparaît que les dispositifs de groupware bénéficient d'un rapport de force qui en fait une modalité de communication incontournable (et parfois même obligatoire); or ces dispositifs sont porteurs d'une symbolique et de caractéristiques techniques et socio-organisationnelles telles qu'ils privilégient en leur sein les communications de travail instrumentales et de type fonctionnel, dirigées vers l'objectif rationnel de l'optimisation des processus productifs liés au travail 
informationnel et communicationnel. L'emphase, mise par les directions sur les qualités d'efficience productive des systèmes d'information et de communication informatisés, et l'énergie et les moyens mis à la disposition de leur «bon usage " privent les autres formes d'interaction d'une partie de leur légitimité, et donc de leur force.

Aller à l'essentiel, grâce à des messages courts, clairs et débarrassés des "scories » de l'échange langagier courant devient alors la norme, tandis que sont stigmatisés par les directions les temps perdus en réunions bavardes, les interminables ou imprévisibles conversations téléphoniques, gênant la concentration de travailleurs intellectuels qui doivent apprendre à gérer au mieux leur efficacité, grâce à une utilisation judicieuse des outils qui permettent l'explicitation, la formalisation, la classification, l'accélération et la fluidification mais aussi la prédictibilité, la traçabilité et la reproductibilité des activités informationnelles et communicationnelles.

\section{Une économie de la relation :}

$31 \mathrm{Au}$ sein du dispositif technico-organisationnel de travail coopératif, les personnes rencontrées distinguent explicitement ce qui, dans le cadre de leur activité, relève de la communication d'informations et ce qui procède de la relation de communication. La première est fonctionnelle et assimilable à un transfert d'informations, qui néanmoins n'est jamais totalement mécanique car il peut être assorti d'un message de niveau d'urgence du délai de réponse attendu, d'éléments explicatifs ou de consignes; il serait d'ailleurs plus juste ici de parler de communication opérationnelle, liée à l'activité professionnelle. La seconde entre aussi sans conteste dans le cadre de l'exercice du métier et possède une dimension instrumentale ; elle est cependant elle aussi clairement assortie d'une forte composante inter-relationnelle, puisqu'il s'agit des échanges grâce auxquels l'inter-subjectivité et l'inter-compréhension s'établissent entre deux sujets (entre des collègues mais aussi entre un collaborateur et un client ou un partenaire externe).

Ce qui apparaît dans les propos et dans le vécu des usages liés à la présence des outils de groupware, notamment dans les deux derniers cas d'étude, c'est une tendance nouvelle pour les salariés à auto évaluer la nature et la pertinence d'une relation de communication, parfois avant même de l'avoir engagée. Du point de vue gestionnaire et managérial. mettre à la disposition des travailleurs des bases d'informations et de connaissances produites et validées par des collaborateurs de l'entreprise et encourager leur utilisation, c'est indiquer qu'il est préférable de se référer à l'existant, capitalisé dans l'entreprise, réutilisable sans frais supplémentaires, et de faire l'économie d'une sollicitation directe à l'égard du collègue compétent. Renoncer à la relation c'est faire preuve d'autonomie dans la recherche d'informations, participer à la politique de valorisation productive des richesses informationnelles de l'entreprise, c'est aussi respecter son collègue et participer à la gestion optima d'un précieux temps de travail. Les dispositifs de groupware sont symboliquement porteurs de tels messages et promus, ouvertement parfois, comme des dispositifs visant prioritairement à gagner du temps, précisément sur celui que l'on consacre aux relations et aux communications de travail. C'est pourquoi les individus intériorisent des attitudes d'autocensure et préfèrent passer parfois plus de temps à rechercher, sur une base informatique, une information qui sera plus ou moins exploitable, plutôt que de solliciter un collègue dont on sait pourtant qu'il pourrait en quelques minutes résoudre une interrogation. 
là un des paradoxes de l'injonction collaborative, qui promeut l'engagement individuel à la production de sens et de pratiques insérés dans le collectif, tout en réduisant cet engagement à l'utilisation de médiateurs techniques. Cette marginalisation de la relation ne se traduit pas forcément par une politique volontaire de raréfaction des rencontres ou des échanges informels mais, si certaines entreprises ont bien compris l'utilité de ces moments et de ces communications informelles et vont jusqu'à recréer des espaces de convivialité ou proposer d'occasionnels séminaires de rencontres conviviales, la tendance semble être celle d'une tentative de dissociation plus forte dans le travail quotidien des parts jugées productives et improductives de l'échange.

$\mathrm{Au}$ final, il s'avère que les dispositifs de groupware, et plus largement les NTIC visant à la médiatisation des communications et des pratiques informationnelles de travail, constituent l'un des instruments de contrôle accru des échanges et sont porteurs d'une visée de gain productivité (qui reste à démontrer). Ils sont aussi et surtout inscrits dans des dynamiques sociales et organisationnelles, portés par (et porteurs) des représentations symboliques et des cadres de pensée structurant une certaine vision instrumentale et fonctionnelle - de la coopération et de la communication de/au travail.

\section{BIBLIOGRAPHIE}

BOUTAUD J.J., Image de soi et organisation. Revue Communication et Organisation, pp. 38-60. GREC/ O. $\mathrm{N}^{\circ}$ 1. mai 1996.

FAGUET-PICQ B., La coopération dans le travail, chap. II : pp. 43-58. in Bollier G. \& Durand C., La nouvelle division du travail, Paris : Editions de l'Atelier, 1999.

FULK J., Social construction of communication technology, Academy of Management Journal, Vol 36 pp. 921-940, Mississipi State, octobre 1993.

FULK J. \& DESANCTIS G. Electronic Communication and Changing Organizational Forms, in Organization Science, pp. 337-349. vol. 6. N 4, juillet/août 1995.

LINHART D., La modernisation des entreprises, Collection Repères. Paris : La Découverte, 1994.

LEPINE V., Les enjeux communicationnels et socio-organisationnels du déploiement de dispositifs de groupware en entreprise. La médiatisation technique du travail collaboratif, Thèse de doctorat en Sciences de la Communication, sous la direction de B. Miège, Université Stendhal-Grenoble 3, décembre 2000.

LEPINE V., Coopération, coordination, collaboration : éléments de définition, contribution aux journées d'études Org \& Co, Aix en Provence, 3-5 juin 1999.

LEVAN K. \& LIEBMAN A., Le groupware. Informatique, management et organisation, collection Systèmes d'information, Paris : Hermès. 1994.

ORLIKOWSKI W., Learning from Notes: Organizational Issues in Groupware Implementation, in Proceedings of the third Conference on CSCW, pp. 362-369, Toronto, novembre 1992. 
PICHAULT F., The Management of Politics in Technically Related Organizational Change, Organizational Studies, pp. 449-476. vol. 16/3. 1995.

ZARIFIAN P., Travail et communication. Essai sociologique sur le travail dans la grande entreprise industrielle, Paris : PUF, 1996.

\section{RÉSUMÉS}

En prenant pour objet les dispositifs de groupware la recherche analyse, du point de vue des SIC, les enjeux liés aux processus de médiatisation technique des communications de travail. Les hypothèses d'une rationalisation du travail intellectuel, d'une coopération "cadrée » et d'une maîtrise accrue des communications sont confrontées à trois cas d'entreprises qui ont mis en place des applications de groupware.

Using the groupware systems as a base, this research conducted in the field of Communication Sciences, analyses the issues concerned with applying technical medias for exchange and communication at work. Three hypothesis are tested in three different companies using groupware systems: the ongoing process of the rationalisation of mental labour, the constrained frame of cooperation, and the raising control of communications.

\section{INDEX}

Mots-clés : coopération, Communications de travail, Groupware, NTIC, organisation

\section{AUTEUR}

\section{VALÉRIE LÉPINE}

Valérie Lépine est titulaire d'un doctorat en Sciences de l'Information et de la Communication de l'Université Stendhal-Grenoble 3, réalisé dans le cadre du Gresec (Groupe de Recherche Sur les Enjeux de la Communication); chercheur au GRESEC et Groupe d'Etudes et de Recherches sur les Communications organisationnelles (Org \& Co) de la Société Française des Sciences de l'Information et de la Communication (SFSIC). 